\title{
Effective Modeling for a Distance-Based Fare Structure with a Time-Expanded Network
}

\author{
Donghyung Yook, Ph.D. \\ Korea Research Institute for Human Settlements \\ Kevin Heaslip, Ph.D., P.E. \\ Virginia Polytechnic Institute \& State University
}

\begin{abstract}
This study addresses the appropriateness of a passenger assignment model for a distance-based fare structure that recently has received attention from transit agencies, along with technological advances in electronic fare payment and global positioning systems. Among the two major passenger assignment models, the study identifies that the schedule-based model has an advantage over the frequency-based model for representing a distance-based fare structure. The schedule-based model that explicitly traces passenger routes enables the accurate calculation of a distance-based fare and its variant. In addition, the study addressed the implementation issues associated with the schedule-based model. As the schedule-based model is considered to require more data than the widely-used frequency-based model, the study suggests a way to mitigate this data requirement issue by approximating the timetable at each stop with the same data used for the implementation of the frequency-based model. A case study on the field application of the schedule-based model and its availability as a ready-to-use option in most commercial software for travel demand modeling is described.
\end{abstract}

\section{Introduction}

Transit fare is a critical factor in transit planning that requires careful consideration from the viewpoints of both transit service providers and users. The major source of revenue for transit agencies is the fare collected from users, which naturally makes agency financial health dependent on fare level and demand. Fares constitutes the majority of the cost of an individual's travel disutility, especially for transit users. Transit fares are out-of-pocket expenses; users are sensitive to fare changes, and such changes can affect ridership. Considering the complicated relationship transit fare has with other variables, it is important 
to determine a fare appropriately according to a transit agency's fare policy and its goals (e.g., maximizing revenues, ridership, welfare) that guide customer-related decisions.

Of several fare structures that govern the fare level, a distance-based fare has been considered by transit agencies recently because of its positive impact on social equity as well as the transit agency's financial health, especially by switching from a flat fare model to a distance-based model. In light of the vertical equity defined by Bullard et al. (2004) that compares mobility need and the ability to cover the needs, a distance-based fare seems to effectively alleviate the disparate impacts caused by a flat fare on low-income and minority households (Farber et al. 2014). Such households often are characterized by short travel distances and high rates of transit usage; thus, the fare they pay may subsidize the cost of other transit user groups under a flat fare structure (Farber et al. 2014). Meanwhile, revenue expectations can increase by structural reform to a distance-based fare, as long-distance transit users are relatively insensitive to fare increases (Daskin et al. 1988). Electronic fare collection technology also fosters the attractiveness of a distance-based fare structure.

Despite efforts to explore its potential, whether a distance-based fare structure is appropriately represented in a planning model or not is in question when the frequency-based model is predominantly used among practitioners. Since a precisely-modeled transportation system provides a "common ground" for examining future system planning (Ortuzar and Willumsen 1994), it is imperative to choose the appropriate modeling scheme to represent the real world. Between the two representation schemes for simulating a transit system - the frequency-based model and the schedule-based model-this paper aims to address the model that is more appropriate for a distance-based fare structure.

The study is organized as follows. The next section discusses the historical perspective of a distance-based fare structure. The subsequent section describes how a distance-based fare structure is differently represented in two passenger assignment models. Next, the implementation issues associated with the schedule-based model, an application example in the field, and implementation in major software packages are addressed. The final section summarizes and concludes the paper.

\section{Past and Present Distance-Based Fare Structure}

In the recent past (the 1960s), a differentiated fare system was used in most cities in the U.S. (Cervero and Wachs 1982). This method gradually was replaced by a flat fare system to provide low-cost transportation to lower-income people as well as for the simplicity of fare collection. Almost all transit agencies in the U.S changed their system to flat fare (Cervero and Wachs 1982). This change became the primary cause of the 1980s transit fiscal crisis.

Cervero (1981) and Cervero and Wachs (1982) attributed the transit agencies' financial difficulties to the insensitivity of the flat fare and suburbanization in 1960s and 1970s. They observed that the suburbanization of the U.S. exacerbated an unhealthy fiscal condition, as many transit operators expanded their service coverage to outlying areas while maintaining their insensitive fare structures. This observation was supported by the comparison of the average mileage between individual bus routes and the total number 
of buses. From 1960 to 1974, the average mileage covered by individual bus routes more than doubled, but total bus mileage declined (Sale and Green 1979).

Although fare structure has gone through changes for many years, and there was a time when differentiated fare structures were widely implemented, once transit agencies adopt a flat fare because of its simplicity, they seem to be unwilling to change to alternatives, regardless of the financial impact. The initial capital costs associated with implementing a differentiated fare structure, such as global position system (GPS) devices and electronic payment systems, contribute to the resistance to change (Yook and Heaslip 2014). However, recent technological developments in fare collection offer transit agencies the opportunity to reconsider these practices. Examples of successful implementation of a distance-based fare structure have been found (TCRP 2003), which can provide transit agencies with the motivation to reconsider the practicality of the structure.

A differentiated fare structure that was difficult to implement in the past now has become a viable option as a result of recent developments in fare collection technologies. In terms of fare media, the increasingly widespread usage of smart cards (TCRP 2003) has made a distance-based fare structure more practical. The smart card's compatibility with multiple operators makes a distance-based fare structure implementable by consolidating transit systems and collecting fares based on the actual distance traveled by passengers. As for the monitoring technology that measures passenger travel distance, GPS is used to perform the tracking. Typically, card readers are installed at controlled entrances such as rail line platforms and buses. In addition, policies regarding technology are being developed to incorporate users' ability to indicate the starting and end points of their trips. By requiring passengers to tap their electronic payment media to card readers at vehicles or on platforms, transit agencies can charge a fare based on passenger usage. With this policy, the travel distance of a trip can be tracked when the passenger boards and exits using a device that identifies the location.

\section{Effectiveness of Schedule-Based Model for Distance-Based Fare Structure}

To model a distance-based fare structure, it is critical to select an appropriate modeling scheme that effectively traces passenger route choices. Since the fare is based on the total passenger in-vehicle distance, a model that closely describes passenger flow is needed for representing the fare structure. This section describes the effectiveness of the schedule-based model for tracing the route choice of a passenger, which enables the precise calculation of a distance-based fare. The explicit tracing of the individual route choice is achievable because all possible time-considered routes, called a time-expanded network, are constructed in the schedule-based model.

In a time-expanded network, all possible ways of the passenger flow are created with virtual links and nodes. Figure 1 shows the conceptual representation scheme of the frequency model and the schedule-based model. As shown, the transit lines used for the frequency-based model are broken down into runs in the schedule-based model, where each run of the transit operation (dotted lines) and the temporal arrival of the runs (end of each arrow) build up all possible ways to the destination. In this structure, the 
network-handling scheme becomes greatly similar to a conventional highway network. A route comprising the sequence of links is replaced by a sequence of runs in the time-expanded network. Tracing passenger route choices is intuitive by following the sequence of virtual links.

For example, in Figure 1, users who depart from the origin at 9:00 AM encounter many choices of vehicle runs as the number of virtual nodes after the temporal arrival of the thick dotted lines. However, they would choose the first or second departing vehicle (indicated by circles) to finish their journey in a time-minimized manner. In this case, if a passenger takes the first arriving vehicle, which is the second run of line 6 , then he/she misses the line 7 bus that departs at 9:20 AM. Taking the second run of bus 5 that connects directly to destination $B$ is the shortest route to the destination, according to the space-time graph.
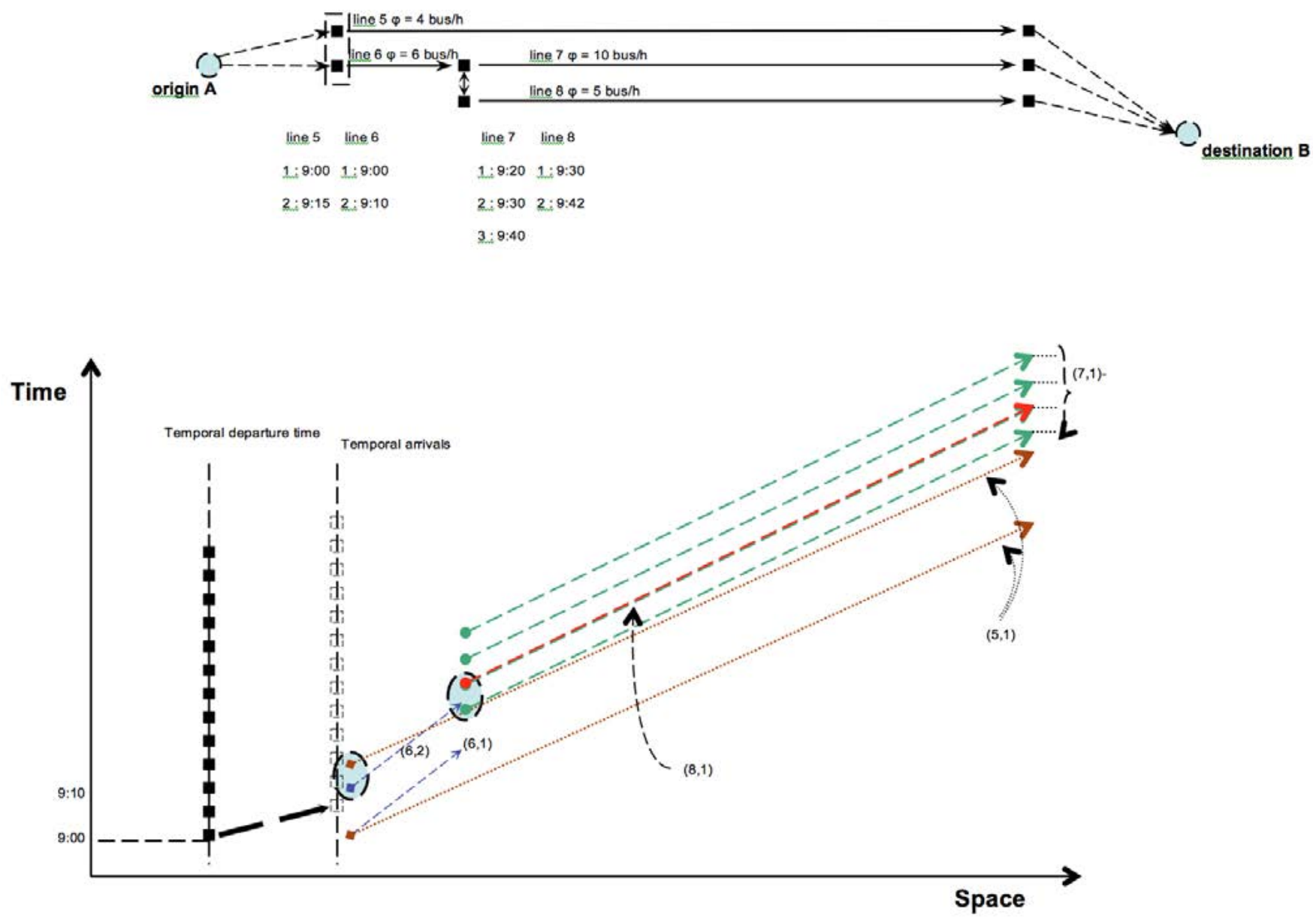

FIGURE 1. Time-expanded network from line-based representation: frequency-based (top) and schedule-based (bottom) 
In the meantime, consideration of the distance-based fare in the frequency-based model is not as flexible as in the schedule-based model. This is because of the line-based representation scheme in which the fare is assessed based on a line segment-the part of a line divided by a transit stop-before a passenger's entire journey is determined. With this modeling scheme, the fare cost of a line segment shared by different routes should be adjusted depending on the routes.

The issue incurred by the frequency-based model for representing a distance-based fare structure is caused by the fact that the fixed fare is used for evaluating the travel cost of a line segment, which does not take into account the possible fare adjustment due to different route compositions. For example, when a different premium is charged for different transit vehicles and the travel distance is compromised by buffer distance, the fare should be assessed only after the route is determined. In this case, the frequency-based model, which considers the fare based on line segment, has a limitation of calculating the accurate distance-based fare. The following paragraphs describe how line-based fare calculation limits the frequency-based model's capability to model the variants of a distance-based fare structure.

The introduction of buffer distance and different premiums for different transit services could produce the following variants of a distance-based fare structure. For the base model and Variant 1, the frequency-based model precisely reflects the actual payment of a user, as the individual fare calculation for a line segment is identical for all routes. On the other hand, a final fare is adjusted at the end of the passenger's journey depending on the routes under Variants 2, 3, and 4. In case of Variants 2 and 4, the total in-vehicle distance is compromised by the buffer distance, which implies that the actual in-vehicle distance subject to fare charge is dependent on the route. However, the absence of route information in the middle of fare calculation for a line segment yields an inconsistent fare evaluation in the frequency-based model.

Figure 2 shows an example of different unit buffer distances applied to each line segment for different line combinations. In this example, a 1-mile buffer distance is applied to Variant 2 , and the buffer distance is divided by the total distance to apply a 1-mile buffer for each line segment. For example, if the total in-vehicle distance is 5 miles, then the buffer distance per mile that amounts to the total 1-mile buffer is 0.2 miles. 
TABLE 1. Variants of Distance-Based Fare Structures

\begin{tabular}{|l|l|l|}
\hline \multicolumn{1}{|c|}{ Fare Model } & \multicolumn{1}{|c|}{ Description } & \multicolumn{1}{c|}{ Formulation } \\
\hline Base Model & Charge fare proportional to total distance & $C_{p}=d_{p} \times u$ \\
\hline Variant 1 & $\begin{array}{l}\text { Charge same base fare for different transit modes and } \\
\text { additional fare proportional to total distance }\end{array}$ & $C_{p}=b+d_{p} \times u$ \\
\hline Variant 2 & $\begin{array}{l}\text { Charge same base fare for different transit modes and } \\
\text { provide buffer distance that base fare covers }\end{array}$ & $C_{p}=b+\left(d_{p}-b u f f e r\right) \times u$ \\
\hline Variant 3 & $\begin{array}{l}\text { Charge different base fares for different transit modes } \\
\text { and additional fare proportional to total distance }\end{array}$ & $C_{p}=\max _{l \in L_{p}}\left[b_{l}\right]+d_{p} \times u$ \\
\hline Variant 4 & $\begin{array}{l}\text { Charge different base fares for different transit modes } \\
\text { and provide buffer distance that base fare covers }\end{array}$ & $C_{p}=\max _{i \in I_{p}}\left[b_{l}\right]+\left(d_{p}-b u f f e r\right) \times u$ \\
\hline
\end{tabular}

where,

$C_{p}=\quad$ distance-based fare of route $p$

$d_{p}=\quad$ in-vehicle distance of route $p$

$u=\quad$ unit fare per unit distance (e.g., mile, kilometer)

$b_{1}=\quad$ base fare for line $l$

buffer $=$ buffer distance

$L_{p}=\quad$ set of lines going through route $p$

There are three line combinations for the base network (top of Figure 2). The numbers above the line indicate the distance. Since one line segment is associated with different routes (in this case, the last 2-mile segment is the last segment for all three line combinations), one fixed unit buffer distance does not exist for Variants 2 and 4. Because the line segment fare calculation is conducted without consideration of route configuration, the fare charge will not be universal for all different routes. For example, when a unit buffer distance of $\$ 0.2$ /mile is assumed, this is appropriate for line combination 2 , yet the value does not properly asses the buffer distance for line combinations 1 and 3 . Since the total route distance for line combinations 1 and 3 are 6 miles and 5.5 miles, respectively, the buffer distance will be 1.2 and 1.1 miles, respectively, which indicates that the actual in-vehicle distance is reduced by more than 1 mile and could result in underestimation of the fare. 

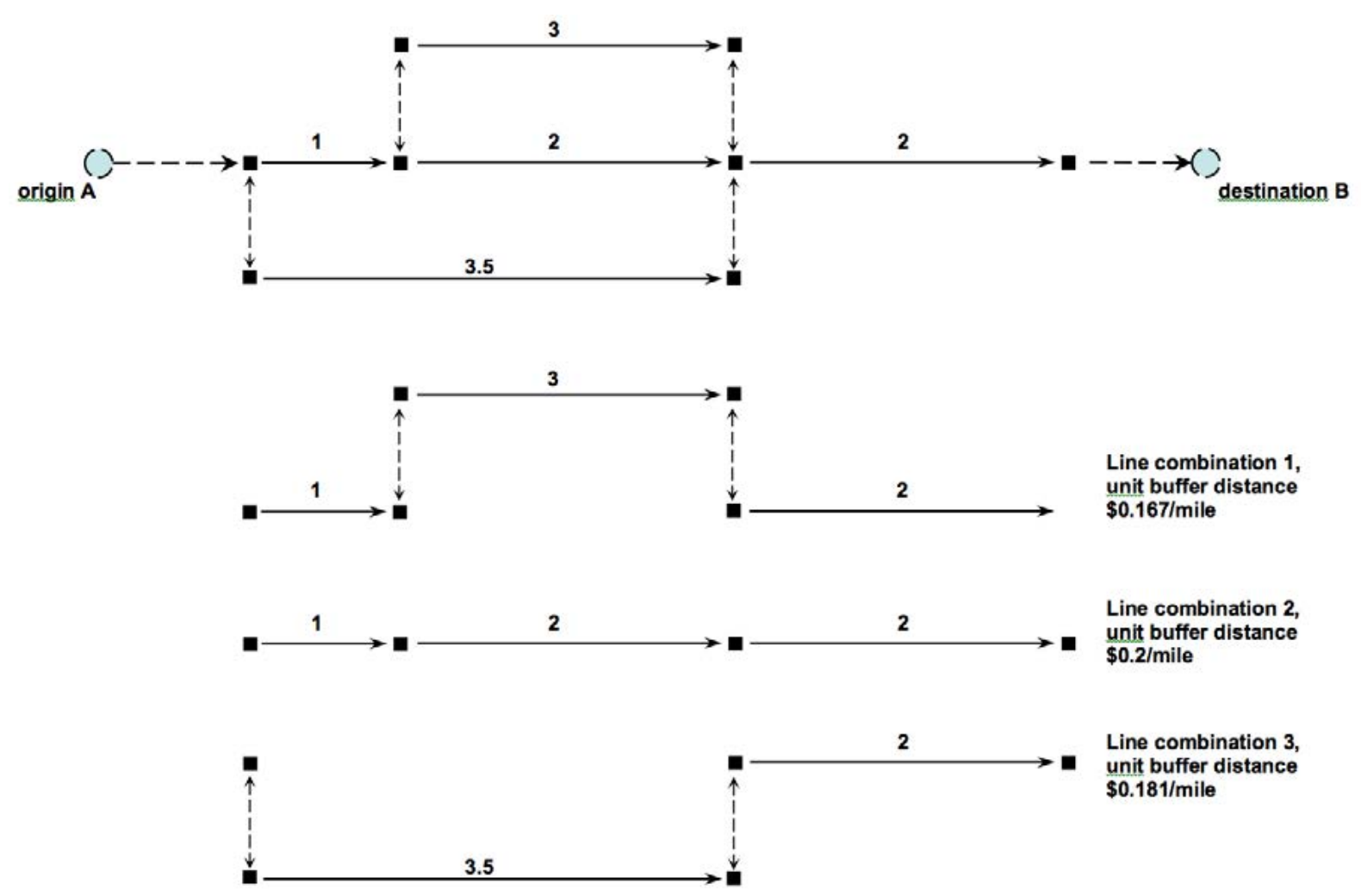

FIGURE 2. Examples of variations of unit buffer distance per mile for different line combinations

A line segment fare calculation independent of route also causes a similar incorrect fare calculation for Variants 3 and 4. In these cases, the maximum premium fare will be charged to the passenger considering his/her vehicle riding history. For example, when a passenger transfers from the first transit vehicle and boards the second vehicle, an additional fare is charged if the second vehicle is premium service (e.g., express bus, commuter rail). This additional fare is dependent on the service level of the second transit vehicle and also the fare already paid when leaving the first transit vehicle. As the frequency-based model does not consider fare adjustment by a combination of different transit vehicles, the model is limited for reflecting the exact fare for a line segment.

The study has identified that the frequency-based model is incapable of representing all variants of a distance-based fare structure. The major factor that hinders the appropriate fare structure modeling is inherited from the line-based service supply system of the frequency-based model. On the other hand, the explicit representation of passenger flow by the schedule-based model can precisely assess all variants of the distance-based fare structure.

In spite of the intuitive feature of the schedule-based model, the modeling scheme has not been widely adopted by transit agencies. The next section focuses on the practical issues of implementing the schedule-based model. 


\section{Implementation}

\section{Data Requirements for Schedule-Based Model}

In the planning stages, the operational details of future transit lines are not provided. This is one reason why the frequency-based model is preferred to the technique based on a time-expanded network. However, with the same level of information given to the frequency-based model, a time-expanded network also can be constructed.

Figure 3 presents typical transit route information for the frequency-based model for a future line in the planning stage. Included are the name of the bus line ("Route 472"), mode (" 6 " means express bus), one-way operation (" $T$ " means true), frequency of the vehicle (30-minute interval), an indicator of the first node (“ $\mathrm{N}$ "), and the route represented by the node sequence.

FIGURE 3.

Sample route information

LINE NAME="ROUTE472", MODE=6, ONEWAY=T, FREQ [1] $=30, N=2108$,

$-1761,-2104,-2102,-2101,-2136,-2098,12642,-2098,-2020,-2018$,

$-2017,-2016,-2014,-1990,-2011,-1957,-2458,1956,-1882,-1881$,

$-1879,-1849,-1853,-1839,-1952,-1837,-1835,-1986,1744,-2554$,

$-1809,-1797,-1807,-9251,-9389,-9409,-9417,-9252,-9434,-9432$.

$-9253,-9254,-9255,-9256,-9258,-9257,-1598,-1600,2522,-1600$,

$-1598,-9259,-9260,-9436,-9438,-9261,-1526,-1528,1530,-1528$,

$-1526,-9262,-9446,-9447,-9448,-9451,-9453,-9201,-10003,-2607$,

$-2623,-9329,-2625,-10377,-10341,-2373,-7746,-10351,-13483,-13485$,

$-10353,-10354,-7272,-7523,-7525,-10358,-10360,-10362,-10357,-7535$,

$-10365,-7526,-10506,-10507,-10374,-10512,-10369,-10246,-3611,-3503$,

$-3663,3531,-3625,-3680,-3688,-3717,3629,-2496,-4861,3631,-3710$,

$-5445,-3638,-5470,-3646,-3651,-3759,-3653,-3654,3655$

With the information shown in Figure 3, a timetable for a time-expanded network can be easily approximated. By estimating the travel time between nodes, the times of the bus arrival at each stop of the future line are calculated. The point of the time approximation is calculated by dividing the distance referred from the highway network by the given operation speed. Then, tagging the start time of the bus operation finalizes the timetable estimation at each stop.

A sample timetable has been constructed with a currently-operating bus route (\#472) of the Utah Transit Authority. Comparison between the approximated timetable and the published timetable is presented in Figure 4. Some deviations are observed from the published timetable at each stop; however, the estimated schedule and the total journey time are well fitted to the published timetable overall. When further information becomes available, a more sophisticated method (Ceder et al. 2013) can be applied for constructing a timetable that minimizes passenger waiting time and number of empty seat hours. 


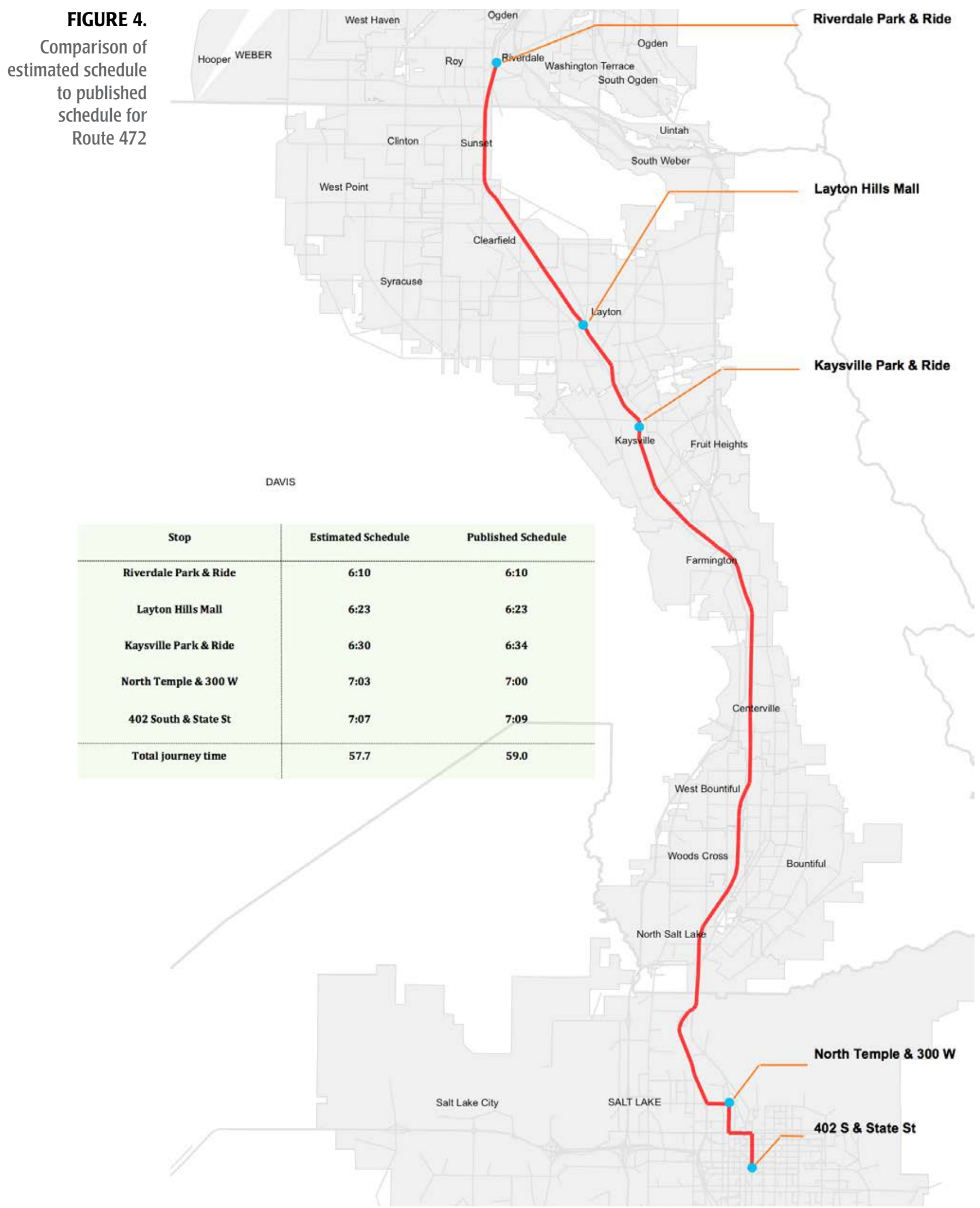




\section{Time Segmented Transit Demand}

Another implementation issue related to the schedule-based model is time-associated demand. Here, the time segmented transit demand means more disaggregated transit demand with a relatively short time interval. As mentioned earlier, the temporal segmentation of transit demand is desirable for its appropriate distributions into a network for the schedule-based model. In general, the travel demand model is framed by peak and non-peak hours, which is longer than two hours.

In this case, it is necessary to disaggregate the demand according to the temporal distribution of demands in the schedule-based model. Segmentation is not a significant issue, since the temporal distribution of travel demand is obtainable from a household travel survey. Determining a correct time interval is more influential for the appropriate distribution of demand into the network. However, densely-segmented demand (less than 15 minutes) can cause large computational burdens due to the large number of temporal zone centroids.

\section{Field Application Cases of Schedule-Based Model}

Field application cases rarely are found despite the rich academic research in the work based on schedule-based transit assignment (Nuzzolo 2003). However, a good pioneer study that models the time dynamics of the supply and demand sides of a transit system with a schedule-based model can be found with TransLink, a transit agency based on South Coast British Columbia, Canada. The study highlights the capability of the model that effectively analyzes the capacity issues of a transit system. The model evaluates the current operating plan's optimality status, identifies future capacity of the critical segment, and determines the fleet strategy for SkyTrain operation. Also, the model helps to examine the impact caused by introducing a new light metro line into adjacent bus routes. The application estimates the ridership change for affected buses and provides an insight for desirable adjustment for the bus route design.

However, the input data considering the demand and supply sides of the transit system that are time-dependent requires considerable effort to construct and validate. As mentioned earlier, the supply side of a transit operation, the time-expanded network, should be prepared using the current operation schedule and fleet management plan. This step amounts to joining the network topology and operation schedule of all transit lines in the study network. The actual challenge for preparing the input data comes from the data construction of demand. Since the schedule-based model necessitates that demand be associated with time, the time-dependent demand should follow the distribution of desired departure times.

This study suggests a simple method to estimate the distribution using a household travel survey. However, if the sample size of the survey is not enough to make a distribution curve, other sources of data such Automated Passenger Count (APC) should be used. This applies to the case of TransLink, in which the departure time distribution was determined from boarding counts. It used four different time frames because the time distribution of the desired departure was different for geographical market segments. As the distance to the Central Business District increased, the peak of the distribution curve shifted to the left on the $x$-axis, representing time, to keep the desired arrival times. Once the time- 
dependent demand is constructed using the time distribution of the desired departure, the demand should be validated with the observed data. In the case of TransLink, the demand was validated by total passenger trips, per mode, per line, and per stop based on the boarding and alighting counts. This validation process was successful for TransLink, as it was equipped with a relatively new, cutting-edge system that produces various aggregated and disaggregated statistics of passenger counts.

This study shows both promising and challenging aspects of the application of the schedule-based model. The promising side contains detailed outputs. As seen in TransLink's case, the schedule-based model is useful for investigating the capacity issue at the planning stage. Considering that the general approach for capacity analysis engages a special process for constructing a load profile based on passenger itineraries, the runbased schedule-based model that simply yields the load profile on a time-expanded network provides a significant advantage. Also, without the modal split process, the schedule-based model estimates user behavioral changes caused by new transit lines, which is sufficient for providing a modification sketch for existing bus route design.

On the other hand, as the well-calibrated data are strongly linked to the validated results, the construction of time-dependent demand is a resource-demanding process. This may be one of the obstacles to agencies adopting the advanced scheme for transit system modeling, especially for those that do not have an APC system.

\section{Schedule-Based Model, a Fully Implementable Option in Commercial Packages}

The major travel demand modeling software, including TransCAD, EMME, Cube, and VISUM, are equipped with the schedule-based assignment model as well as the frequency-based model. Since the schedule-based model needs a time-expanded network for modeling the supply side of the transit system, timetable information associated with spatial route data is necessary. Similar to the suggested simple method that estimates the timetable at each stop, most software provide an internal mechanism to estimate the running time between stops using the given highway network or transit operation speed. In the case of VISUM, running time is calculated from the quotient of link length and link-specific speed (VISUM 2011), whereas EMME uses results from the evaluation of transit time functions (Florian 1999). When analysts use this scheme, no extra information is required other than the conventional input data, as presented in Figure 3 for the frequency-based model.

However, considering the time dynamics of the schedule-based model, if the highway network or the transit time function are not sophisticated enough to represent the temporal difference associated with each run of a transit vehicle, it is highly likely that discrepancies will be produced between the published timetable and the estimated timetable. It is recommended to examine the estimated running time based on the published timetable to minimize errors from transit operation modeling.

Most software provides supplemental tools to modify the estimated running time by means of a modification dialog box or a dedicated coding system. Table 2 shows the transit segment time coding scheme provided by EMME/2. When using the first 10 nodes of the sample route information given in Figure 3 , the timetable coding is generated as shown in Table 2. 
TABLE 2.

Example of Timetable Coding Used in EMME/2

\begin{tabular}{|c|c|c|}
\hline Node & Run1 & Run2 \\
\hline 2108 & 7h00 & 7h20 \\
\hline 2102 & 7h15 & 7h35 \\
\hline 12642 & 7h42 & 8h02 \\
\hline
\end{tabular}

Since the node number followed by negative sign is not a stop, only a stop is tagged with published departure time; the coding scheme is straightforward.

\section{Summary and Conclusions}

Although a distance-based fare structure is regarded to achieve fairness and equity in fare charging, it has not been widely adopted by transit agencies. However, recent technological developments in electronic fare payment systems and GPS devices create a favorable condition for implementing a distance-based fare structure.

Following advancements in fare payment systems, the study investigated the appropriateness of passenger assignment models for a distance-based fare structure. Among the two major passenger assignment models, the study identified that the schedule-based model is more suitable for representing a distance-based fare structure. The schedule-based model explicitly traces passenger route and enables accurate calculation of a distance-based fare and its variants, whereas the frequency-based model has limited capability for handling all variations of a distance-based fare structure due to its line segment-based fare calculation method. In addition, the study addressed implementation issues for the schedule-based model. Even if the data requirements for the schedule-based model are higher than its counterpart, the study suggested a simple method that reduces the data requirements and explained the method as a ready-to-use option for most commercial travel demand modeling software.

\section{References}

Bullard, R.D., G. S. Johnson, and A. O. Torres. 2004. Highway Robbery: Transportation Racism \& New Routes to Equity. Cambridge, MA: South End Press.

Ceder, A., S. Hassold, C. Dunlop, and I. Chen. 2013. Improving urban public transport service using new timetabling strategies with different vehicle sizes. International Journal of Urban Sciences, 17: 239-258.

Cervero, R. 1981. Flat versus differentiated transit pricing: What's a fair fare? Transportation 10: $211-232$.

Cervero, R., and M. Wachs. 1982. An answer to the transit crisis: The case for distancebased fares. Journal of Contemporary Studies, 5: 59-70.

Chu, K. K. A., and R. Chapleau. 2008. Enriching archived smart card transaction data for transit demand modeling. Transportation Research Record, 2063: 63-72. 
Daskin, M. S., J. L. Schofer, and A. E. Haghani. 1988. A quadratic programming model for designing and evaluating distance-based and zone fares for urban transit. Transportation Research Part B, 22: 25-44.

De Cea, J., and E. Fernandez. 1993. Transit assignment for congested public transport systems: An equilibrium model. Transportation Science, 27: 133-147.

Farber S., K. Bartholomew, X. Li, A. Paez, and K. M. Nurul Babib. 2014. Assessing social equity in distance based transit fares using a model of travel behavior. Transportation Research Part A, 67: 291-303.

Florian, M. 1999. Deterministic time table transit assignment. First Asian EMME/2 Users Group Meeting, Shanghai, P.R. China.

Lampkin, W., and P. D. Saalmans. 1967. The design of routes, service frequencies and schedules for municipal bus undertaking: A case study. Operational Research Quarterly, 18: 375-397.

Nuzzolo, A. 2003. Transit path choice and assignment model approaches. In Lam, W. H. K., and M. G. H. Bell (eds.), Advanced Modeling for Transit Operations and Service Planning. Oxford: Pergamon.

Ortuza, J. D., and L. G. Willumsen. 1994. Modeling Transport. New York: John Wiley \& Sons.

Sale, J. E., and B. Green. 1979. Operating costs and performance of American public transit systems. Journal of the American Planning Association, 45: 22-27.

Transit Cooperative Research Program (TCRP). 1996. Fare policies, structure and technologies. Transportation Research Board, Washington DC, Report 10, Chapter 3.

TCRP. 2003. Fare policies, structure and technologies: Update. Transportation Research Board, TRCP Report 94, Chapter 2.

Tong, C. O., and S. C. Wong. 1999. A schedule-based time-dependent trip assignment model for transit networks. Journal of Advanced Transportation, 33: 371-388.

VISUM manual, 2011. VISUM 11.52 - Fundamentals. PTV AG, Karlsruhe Germany.

Yook, D., and K. Heaslip. 2014. Determining appropriate fare levels under distancebased fare structure considering users' behaviors in a time-expanded network, Transportation Research Record, 2415: 127-135.

\section{About the Authors}

DoNGHYUNG Yook, Ph.D. (dhyook7@aggiemail.usu.edu) is an Associate Research Fellow at the Korea Research Institute for Human Settlements. He is a graduate of the doctoral transportation engineering program at Utah State University.

Kevin HeAslip, Ph.D., P.E. (kheaslip@vt.edu) is an Associate Professor of Civil \& Environmental Engineering at Virginia Tech's Arlington Research Center. He previously was an Associate Professor at Utah State University. 